\title{
Oncolytic vaccinia virus delivering tethered IL-12 enhances antitumor effects with improved safety
} Yan Ge,,$^{1,2,3}$ Haiyan Wang,,$^{1,2,3}$ Jinghua Ren, ${ }^{1,2,4}$ Weilin Liu, $^{1,2,5}$ Lingjuan Chen, ${ }^{1,2,4}$
Hongqi Chen, ${ }^{1,2,6}$ Junjie Ye, ${ }^{1,2,7}$ Enyong Dai, ${ }^{1,2,8}$ Congrong Ma, $^{1,2}$ Songguang Ju, ${ }^{3}$
Zong Sheng Guo, ${ }^{1,2}$ Zuqiang Liu, ${ }^{1,2}$ David L Bartlett
To cite: Ge Y, Wang H, Ren J, et al. Oncolytic vaccinia virus delivering tethered IL-12 enhances antitumor effects with improved safety. Journal for ImmunoTherapy of Cancer 2020;8:e000710. doi:10.1136/ jitc-2020-000710

- Additional material is published online only. To view please visit the journal online (http://dx.doi.org/10.1136/jitc2020-000710).

YG and HW contributed equally.

ZL and DLB are joint senior authors.

Accepted 26 February 2020

Check for updates

(c) Author(s) (or their employer(s)) 2020. Re-use permitted under CC BY-NC. No commercial re-use. See rights and permissions. Published by BMJ.

For numbered affiliations see end of article.

Correspondence to

Dr Zuqiang Liu; liuz@upmc.edu

Dr David L Bartlett;

bartdl@UPMC.EDU

\section{ABSTRACT}

Immune checkpoint blockade is arguably the most effective current cancer therapy approach; however, its efficacy is limited to patients with "hot" tumors, warranting an effective approach to transform "cold" tumors. Oncolytic viruses (especially properly armed ones) have positive effects on almost every aspect of the cancer-immunity cycle and can change the cancerimmune set point of a tumor. Here, we tested whether oncolytic vaccinia virus delivering tethered interleukin 12 (IL-12) could turn a "cold" tumor into a "hot" tumor while avoiding IL-12's systemic toxicity. Our data demonstrated that tethered IL-12 could be maintained in the tumor without treatment-induced toxic side effects. Moreover, the treatment facilitated tumor infiltration of more activated $\mathrm{CD}^{+}$and $\mathrm{CD}^{+} \mathrm{T}$ cells and less Tregs, granulocytic myeloid-derivedsuppressor cells, and exhausted CD8 ${ }^{+}$ $T$ cells, with increased interferon $\gamma$ and decreased transforming growth factor $\beta$, cyclooxygenase-2, and vascular endothelial growth factor expression, leading to transformed, immunogenic tumors and improved survival. Combined with programmed cell death 1 blockade, vaccinia virus expressing tethered IL-12 cured all mice with late-stage colon cancer, suggesting immediate translatability to the clinic.

\section{INTRODUCTION}

Cancer immunotherapy is joining surgery, chemotherapy, and radiotherapy as a standard treatment modality in the war against cancer. It relies on tipping the cancer-immune set point ${ }^{1}$; that is, changing the equilibrium between factors that stimulate or inhibit anticancer immunity to restore anticancer effects. These tumor cell-intrinsic or cellextrinsic factors within the tumor microenvironment (TME) are coordinated at each step of the cancer-immunity cycle. ${ }^{2}{ }^{3}$ Immune checkpoint blockade is arguably the most important approach during the past decade to modulate the cancer-immunity cycle for cancer therapy. Such blockades include application of antibodies to target the cytotoxic T-lymphocyte antigen 4 (CTLA-4)/ CD28 ${ }^{4}$ and programmed cell death 1 (PD-1)/
PD-ligand 1 (PD-L1) axes. ${ }^{5}$ While dramatic, durable, and therapeutic responses are observed after immune checkpoint inhibitor therapy, these successes are still limited to a small percentage of patients. ${ }^{6}$ This is largely attributed to the lack or paucity of $\mathrm{T}$ cell infiltration in most tumors, defining the so-called non-T-cell-inflamed or "cold" tumors. ${ }^{7}$ Therefore, approaches that can convert non-T-cellinflamed or "cold" tumors into T-cell-inflamed or "hot" tumors, mainly characterized by an abundance of tumor-infiltrating $\mathrm{T}$ cells, are urgently needed.

Oncolytic viruses can selectively replicate in and destroy tumor cells and stromal cells while exposing antigens for crosspresentation and elucidating danger signals, triggering innate and adaptive immunity. ${ }^{8}$ Oncolytic viruses have positive effects on almost every aspect of the cancer-immunity cycle and can be further armed with chemokines to improve tumor $\mathrm{T}$ cell infiltration; with cytokines to reprogram immune cells; or with ligands or antibodies to block immune checkpoints and relieve immunosuppression. ${ }^{9}$ Thus, oncolytic viruses might be perfect candidates to modulate the TME and convert "cold" tumors into "hot" tumors. Cytokines have gained researchers' continuous interest for improving the therapeutic effects of oncolytic viruses. ${ }^{10}$ However, uncontrolled cytokine expression and secretion by a live, replicating virus may lead to toxicity and cytokine release syndrome. Recently, we generated an oncolytic vaccinia virus expressing membrane-bound interleukin 2 (IL-2) to diminish the severe toxicity associated with systemic IL-2 exposure and to modulate the TME. This virus not only reduced toxic side effects but also tipped the cancer-immune set point in the TME and treated several murine tumor models. In combination with PD-1/PD-L1 blockade, this virus cured most 
of the mice with a high tumor burden. ${ }^{11}$ IL-12 is another cytokine with great expectations for cancer therapy based on the therapeutic success of IL-12 treatment in a variety of murine tumor models. ${ }^{12}$ However, these expectations have been greatly diminished because systemic IL-12 in clinical trials evoked severe side effects such as leukopenia and thrombocytopenia. ${ }^{13-15}$ Several IL-12 modifications have been explored to improve its safety while preserving its activity. ${ }^{16-21}$ The goal of this study was to investigate whether and how an oncolytic vaccinia virus expressing membrane-bound IL-12 modulates the TME and further improves antitumoral effects alone or in combination with immune checkpoint blockade.

\section{RESULTS AND DISCUSSION}

To reduce the severe toxic side effects caused by the systemic application of IL-12, we used vvDD, a double viral gene-deleted ( $t k^{-}$and $v g f-$ ) vaccinia virus, to deliver membrane-bound IL-12 into the tumor bed. This replicating virus is tumor-selective ${ }^{22}$ and its safety has been demonstrated in clinical trials. ${ }^{23}{ }^{24}$ We constructed vvDDIL-12, vvDD-IL-12-FG, and vvDD-IL-12-RG to express secreted IL-12 or membrane-bound IL-12 after infection in tumor cells, respectively. Membrane association is realized by the glycosylphosphatidylinositol (GPI) anchor form of human CD16b. The difference between vvDD-IL12-FG and vvDD-IL-12-RG is that between the IL-12p40 subunit and GPI anchor, the former has a flexible linker $\left(\mathrm{G}_{4} \mathrm{~S}\right)_{3}$ and the latter has a rigid linker $\left(\mathrm{A}\left(\mathrm{EA}_{3} \mathrm{~K}\right)_{4} \mathrm{AAA}\right)^{25}$ (online supplementary figure S1). When MC38-luc, AB12-luc, and B16 cells were infected with these three IL-12-armed viruses or control virus vvDD at a multiplicity of infection (MOI) of 1 , viral housekeeping gene (A34R) mRNA levels were similar among the viruses, consistent with similar in vitro infection and replication of the different viruses. In addition, the IL-12 mRNA levels were similar in IL-12-armed viruses, as expected (figure 1A). We also measured IL-12 expression at the protein level
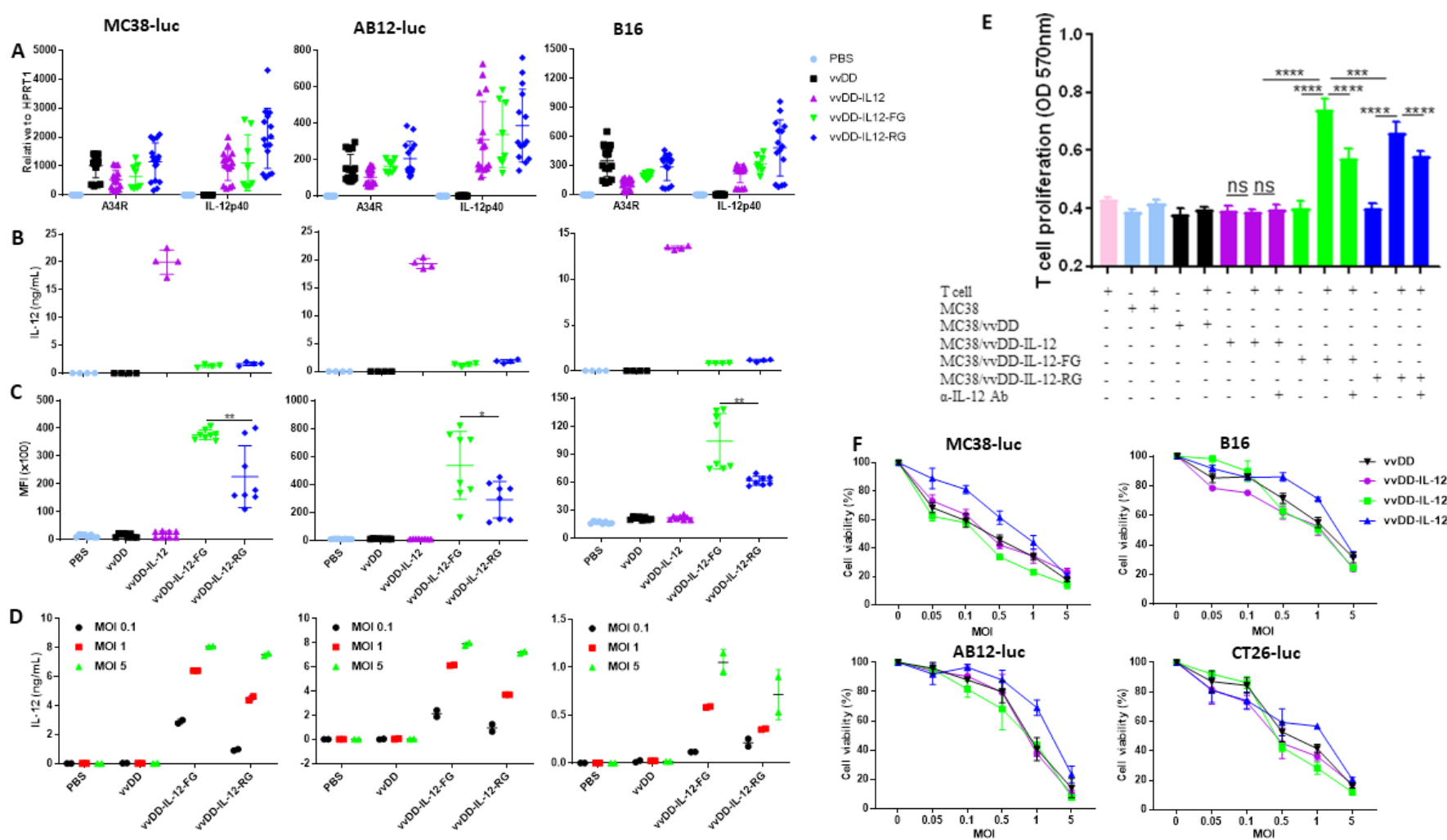

Figure 1 Tethered IL-12 variants show functional IL-12 membrane association and similar cytotoxicity. (A) Tumor cell MC38luc $\left(3 \times 10^{5}\right.$ cells $), B 16\left(2 \times 10^{5}\right.$ cells $)$, or AB12-luc $\left(3 \times 10^{5}\right.$ cells) were mock-infected or infected with vVDD, vVDD-IL-12, vvDDIL-12-FG, or vvDD-IL-12-RG at an MOI of 1. The cell pellets were harvested to measure A34R or IL-12 expression at 24 hours using RT-qPCR. The culture supernatants were harvested to measure secreted IL-12 using ELISA (B) and the cell pellets were also harvested to measure membrane-bound IL-12 using flow cytometry (cell surface staining) (C) 24 hours post-infection. (D) MC38-luc $\left(3 \times 10^{5}\right.$ cells), B16 $\left(2 \times 10^{5}\right.$ cells $)$, or AB12-luc $\left(3 \times 10^{5}\right.$ cells) were mock-infected or infected with vVDD, vvDD-IL-12, vvDD-IL-12-FG, or vvDD-IL-12-RG at MOls of $0.1,1$, and 5. The cell pellets were harvested to measure membrane-bound IL-12 using ELISA after PI-PLC cleavage 24 hours post-infection. (E) Naïve B6 splenocytes were activated and stimulated with IL-12 variant-infected MC38 cells (Responder: stimulator=1:5) in the absence/presence of $\alpha-I L-12 \mathrm{Ab}$, and T cell proliferation was

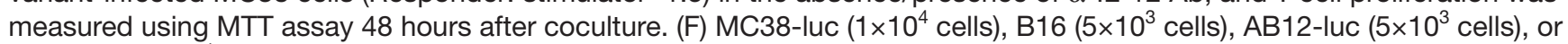
CT26-luc $\left(1 \times 10^{4}\right.$ cells) were infected with IL-12 variants at indicated MOls and cell viability was measured using Cell Counting Kit-8 or MTS assay 48 hours post-infection. Data represent two independent experiments. ${ }^{*} \mathrm{P}<0.05$; ${ }^{\star \star} \mathrm{P}<0.01$; ${ }^{* \star *} \mathrm{P}<0.001$; and ${ }^{\star \star \star *} \mathrm{P}<0.0001$. MOI, multiplicityof infection; NS, not significant; PI-PLC, phosphatidylinositol-specificphospholipase C. 
using ELISA and flow cytometry. The amount of IL-12 in the supernatant from vvDD-IL-12-infected tumor cells was significantly higher than the other constructs (figure 1B), while cell-surface IL-12 expression was significantly more prevalent in vvDD-IL-12-FG-infected or vvDD-IL-12-RGinfected cells (figure 1C; online supplementary figure S2). This demonstrates the successful realization of membrane association by GPI anchored to one subunit of IL-12. This was further confirmed by measuring the amount of IL-12 cleaved by phosphatidylinositol-specific phospholipase C (PI-PLC) from membrane-associated GPI-anchored IL-12. As expected, membrane-bound IL-12 correlated with virus MOI (figure 1D). We further demonstrated that the GPI-anchored IL-12 is functional in vitro. Con A-activated mouse splenic T cells were co-cultured with mitomycin C (MMC)-inactivated MC38 cells, which were mock-infected or infected with the viruses overnight and washed thoroughly with phosphatebuffered saline (PBS) before co-culture. Only wDD-IL12-FG-infected or vvDD-IL-12-RG-infected MC38 cells which had membrane-associated IL-12 could stimulate the proliferation of activated $\mathrm{T}$ cells. This stimulation was significantly attenuated by IL-12 antibody neutralization (figure 1E). In a previous report, a single-chain IL-12 fused with GPI anchor from human folate receptor and a flexible linker from pyruvate ferredoxin oxidoreductase was used for membrane association; however, the percentage of IL- $12^{+}$cells was moderate and a large amount of IL-12 was released into the culture supernatant after adenovirus delivery. ${ }^{17}$ This indicates that the delivery vector, GPI anchor sequence, and linker sequence might affect the membrane association of GPI-anchored proteins. In fact, we previously showed that a rigid linker between the IL-2 and GPI anchor is better for IL-2 membrane association than a flexible linker, ${ }^{11}$ whereas here a flexible linker was better for IL-12 membrane association suggesting an effect of the nature of targeted proteins on the design of a GPI-anchored protein. The cytotoxicity of IL-12-armed viruses was tested in four murine tumor cells. The results demonstrated that they had similar in vitro cytotoxicity compared with the parental virus voD (figure 1F).

To investigate the toxicity induced by these viruses, we first measured the levels of IL-12 and interferon $\gamma($ IFN- $\gamma$ ) in mouse sera and tumor nodules. The IL-12 levels were significantly higher in sera from mice treated with voDDIL-12 compared with those treated with membrane-bound forms (online supplementary figure S3A); however, as a main mediator of IL-12-induced antitumor effects, ${ }^{26}$ IFN- $\gamma$ levels in sera were similar after the different IL-12armed virus treatments (online supplementary figure S3B). Therefore, membrane-bound IL-12 might have a similar function with a lower risk of toxicity. The levels of either IL-12 or IFN- $\gamma$ in tumor nodules receiving vvDDIL-12-FG treatment were significantly higher compared with other treatments (figure 2A,B). We next investigated the membrane association of IL-12 in vivo using flow cytometry. There were significantly more IL $-12^{+}$cells in the tumor in mice receiving vvDD-IL-12-FG treatment than in those receiving other treatments (online supplementary figure S3C). This further confirmed that vvDDIL-12-FG could retain IL-12 at the tumor site, compared with other constructs. We also found that only vwDD-IL-12 treatment $\left(1 \times 10^{9}\right.$ plaque-forming units $(\mathrm{PFU}) /$ mouse $)$ induced lung and kidney edema as evidenced by an increase in water content in lungs and kidneys after treatment (figure 2C,D), and liver toxicity as evidenced by an increase in aspartate transaminase (AST) and alanine transaminase (ALT) in the sera (figure 2E,F). While the mice tolerate doses up to $1 \times 10^{9} \mathrm{PFU}$ and primates have been shown to be more permissive to WR strain vaccinia virus infection, ${ }^{27}$ we anticipate that a similar dose expressing membrane-tethered IL-12 may be potent in humans. Collectively, our data demonstrate that vvDDIL-12-FG can effectively maintain IL-12 in the TME and is therefore safer. Hence, we selected vvDD-IL-12-FG for further investigation in the remainder of this study.

To evaluate the antitumoral efficacy of voDD-IL12-FG, we intraperitoneally injected virus at the dose of $2 \times 10^{8} \mathrm{PFU} /$ mouse to treat $\mathrm{B} 6$ mice bearing 5 -day-old peritoneal murine colon cancer (MC38-luc). Survival results demonstrated that vvDD-IL-12-FG and vvDD-IL-12 elicited potent antitumoral effects compared with PBS or vvDD treatment (figure 2G). Impressively, vvDD-IL-12-FG treatment cured all mice that received the treatment, though there was no significant difference in survival between the different IL-12-armed virus treatments. All the mice bearing peritoneal MC38-luc cured by vvDD-IL-12-FG treatment received a subcutaneous re-challenge of either MC38 or an irrelevant tumor control, Lewis lung cancer (LLC). MC38 tumor growth was retarded in the cured mice (figure 2H), but LLC growth was not (figure 2I), compared with naive mouse control, suggesting that a systemic tumor-specific antitumor immunity was elicited. We explored the therapeutic efficacy of voD-IL12 -FG using $2 \times 10^{8}$ or $1 \times 10^{8} \mathrm{PFU} /$ mouse to treat BalB/c mice bearing 5-day-old peritoneal murine colon cancer (CT26-luc; online supplementary figure S3D) or murine mesothelioma (AB12-luc; online supplementary figure S3E) with similar results, respectively. We also evaluated the antitumoral efficacy of vvDD-IL-12-FG in 9-day tumorbearing mouse models, which are more akin to metastatic human tumors, characterized by heavier tumor burden and increased immunosuppressive factor expression in the TME (PD-1, PD-L1, CTLA-4, transforminggrowth factor $\beta$ (TGF- $\beta$ ), CD105, and vascular endothelial growth factor (VEGF)). ${ }^{11}$ Both IL-12-armed virus treatments significantly improved survival compared with PBS or vvDD treatment in this 9-day MC38 model (figure 2J). The splenocytes from 9-day MC38-bearing mice receiving IL-12-armed virus treatment secreted significantly more IFN- $\gamma$ after restimulation with MMC-inactivated MC38, suggesting systemic antitumor immunity in the latestage tumor model (figure 2K). Similar survival results were obtained using a 9-day AB12-bearing mouse model (figure 2L). Occasionally, a few mice that received vvDDIL-12 treatment, but not vvDD-IL-12-FG treatment, died 

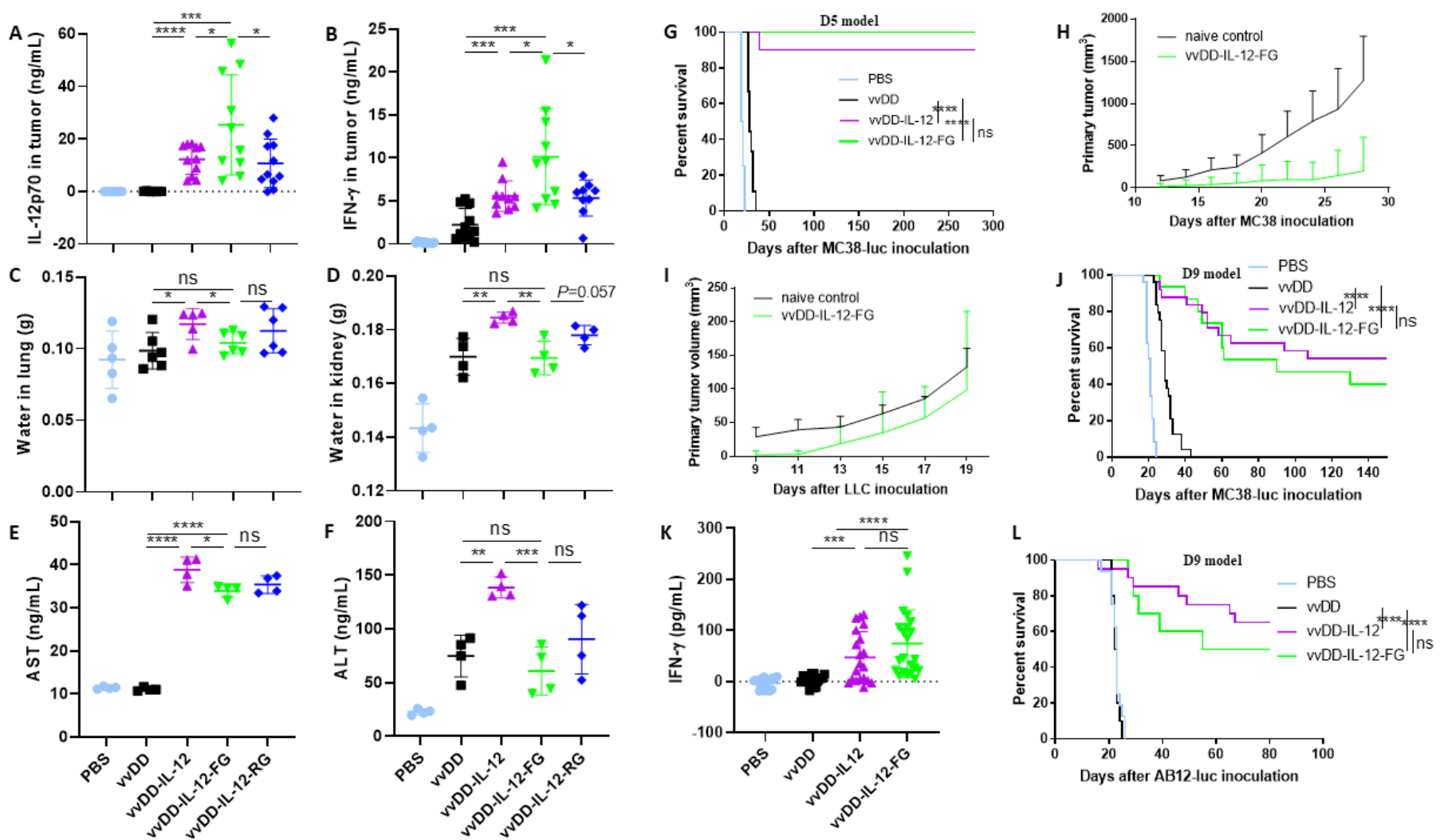

Figure 2 vvDD-IL-12-FG treatment produces tethered IL-12 in tumors and is safe and effective in therapeutic tumor models. B6 mice were intraperitoneally inoculated with $5 \times 10^{5}$ MC38-luc cells and treated with PBS, vvDD, vvDD-IL-12, vvDD-IL-12-FG, or vvDD-IL-12-RG at $1 \times 10^{9} \mathrm{PFU} /$ mouse 9 days post-tumor inoculation. The mice treated above were sacrificed at day 5 after treatment. Tumor nodules were collected to measure the amount of IL-12 (A) and IFN- $\gamma$ in tumor (B); the lungs and kidneys were collected to monitor pulmonary tissue edema (C and D); sera were collected to measure AST (E) and ALT (F) in sera. (G) B6 mice were intraperitoneally inoculated with $5 \times 10^{5}$ MC38-luc cells and treated with PBS, vvDD, vvDD-IL-12, or vvDD-IL-12-FG at $2 \times 10^{8} \mathrm{PFU} /$ mouse 5 days post-tumor inoculation $(\mathrm{n} \geq 8)$. The vVDD-IL-12-FG cured mice were subcutaneously re-challenged with MC38 $(\mathrm{H})$ or LLC (I). B6 mice were intraperitoneally inoculated with $5 \times 10^{5}$ MC38-luc cells and treated with PBS or indicated viruses at $2 \times 10^{8}$ PFU/mouse 9 days post-tumor inoculation $(n \geq 23)$. The survival curve was shown (J). Some of these treated mice were sacrificed and splenocytes were restimulated with mitomycin C-inactivated MC38 cells to monitor IFN- $\gamma$ production (K). (L) BALB/c mice were intraperitoneally inoculated with $4 \times 10^{5} \mathrm{AB} 12$-luc cells and treated with PBS or indicated viruses at $2 \times 10^{8} \mathrm{PFU} / \mathrm{mouse} 9$ days post-tumor inoculation ( $\mathrm{n} \geq 10$ ). A log-rank (Mantel-Cox) test was used to compare survival rates. ${ }^{\star} \mathrm{P}<0.05 ;{ }^{* \star} \mathrm{P}<0.01$; ${ }^{* \star} \mathrm{P}<0.001$; and ${ }^{\star \star * \star} \mathrm{P}<0.0001$. ALT, alanine transaminase; AST, aspartate transaminase; IFN- $\gamma$, interferon $\gamma$; IL-12, interleukin 12; NS, not significant; PBS, phosphate-buffered saline; PFU, plaque-forming units.

earlier than those that received PBS treatment (figure 2L), consistent with the IL-12-induced toxicity.

To explore the mechanism by which voDD-IL-12-FG treatment elicits antitumoral immune activity in the profoundly immunosuppressive advanced tumor model, we investigated the immune cell profile in the TME using the late-stage tumor model. The percentages of $\mathrm{CD} 4^{+} \mathrm{Foxp}^{-}$and $\mathrm{CD} 8^{+} \mathrm{T}$ cells from tumors receiving IL-12-armed virus treatment were increased compared with those treated with PBS or vvDD (figure 3A,B) and the IFN- $\gamma$ secretion from both $\mathrm{CD}^{+}$and $\mathrm{CD}^{+} \mathrm{T}$ cells was increased (figure $3 \mathrm{C}, \mathrm{D}$ ). The results also showed that the exhausted $\mathrm{PD}^{+} \mathrm{CD}^{+} \mathrm{T}$ cells, more severely exhausted $\mathrm{PD}^{+} \mathrm{Tim}^{-} 3^{+} \mathrm{CD} 8^{+}, \mathrm{PD}^{+} \mathrm{TIGIT}^{+} \mathrm{CD} 8^{+}$, and $\mathrm{PD} 1^{+} \mathrm{LAG}-3^{+} \mathrm{CD} 8^{+} \mathrm{T}$ cells in the tumor-infiltrating $\mathrm{CD} 8^{+}$ T-cell population decreased after IL-12-armed virus treatment (figure 3E-H). We examined myeloid-derived suppressor cells (MDSCs) in tumors after virus treatment. As reported by a previous study, ${ }^{28}$ we found that granulocytic MDSCs (G-MDSCs, CD11b ${ }^{+} \mathrm{Ly}_{6 \mathrm{G}^{+}} \mathrm{Ly}_{6 \mathrm{C}^{\text {low }}}$ ) were increased after vvDD treatment; however, they were decreased after IL-12-armed virus treatment (figure 3I). We also examined regulatory T cells (Tregs, CD $4^{+}$Foxp $3^{+}$) and found that the percentage of $\mathrm{CD} 4^{+} \mathrm{Foxp}^{+} \mathrm{T}$ cells in tumor-infiltrating $\mathrm{CD} 4^{+} \mathrm{T}$ cells was decreased after IL-12armed virus treatment (figure 3J), implying suppression of tumor-induced Treg proliferation by the IL-12/ IFN- $\gamma$ axis, ${ }^{29}$ owing to the significantly higher IL-12 and IFN- $\gamma$ levels in tumors that received IL-12-armed virus treatments (figure 2A,B; online supplementary figure $\mathrm{S} 4 \mathrm{~A})$. However, the increase of IFN- $\gamma$ in the TME did not upgrade the expression of $\mathrm{PD}-1$ on $\mathrm{T}$ cells (figure $3 \mathrm{E}$; online supplementary figure S4B-C) or PD-L1 on CD45

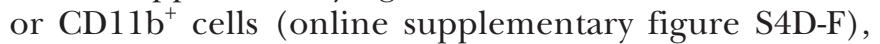
suggesting that IL-12 did not reinforce adaptive immune resistance in vvDD-related therapy. ${ }^{30}$ We next found a significant decrease in the expression of pro-cancer factors, including TGF- $\beta$, cyclooxygenase-2 (COX-2), 

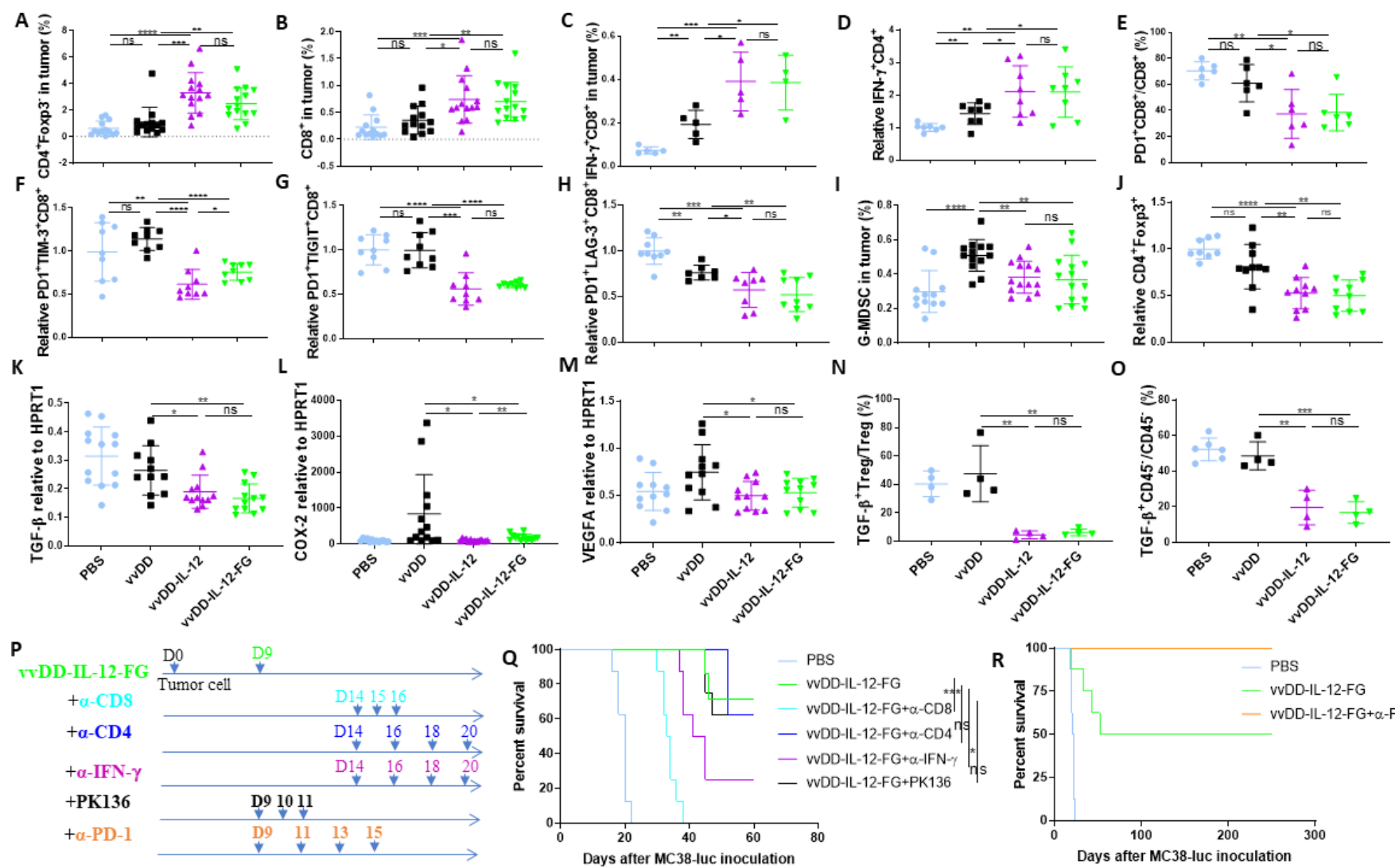

Figure 3 IL-12-variant treatments modify the tumor microenvironment and improve the therapeutic effect with PD-1 blockade. B6 mice were intraperitoneally inoculated with $5 \times 10^{5} \mathrm{MC} 38$-luc cells and treated with PBS, vvDD, vvDD-IL-12, or vvDD-IL-12FG at $2 \times 10^{8} \mathrm{PFU} /$ mouse 9 days post-tumor inoculation. Tumor-bearing mice were sacrificed 5 days post-treatment and primary tumors were collected and analyzed using flow cytometry to determine $\mathrm{CD} 4^{+} \mathrm{Foxp} 3^{-}(\mathrm{A})$ and $\mathrm{CD} 8^{+} \mathrm{T}$ cells $(\mathrm{B}), \mathrm{IFN}-\gamma^{+} \mathrm{CD} 8^{+}(\mathrm{C})$, IFN- $\gamma^{+} \mathrm{CD}^{+}(\mathrm{D})$, exhausted CD8 ${ }^{+}$T cell $(\mathrm{E}-\mathrm{H})$, G-MDSCs (I), or regulatory T cells $\left(\mathrm{CD} 4^{+} \mathrm{Foxp} 3^{+}\right)(\mathrm{J}), \mathrm{TGF}^{+} \beta^{+} \mathrm{Treg}(\mathrm{N}), \mathrm{TGF}^{+} \beta^{+} \mathrm{CD} 45^{-}$ $(\mathrm{O})$, using RT-qPCR to determine TGF- $\beta$, COX-2, and VEGF (K-M). In a separate experiment, B6 mice were intraperitoneally inoculated with $5 \times 10^{5} \mathrm{MC} 38$-luc cells and treated with vvDD-IL-12-FG or PBS 9 days post-tumor inoculation. Anti-CD8 Ab

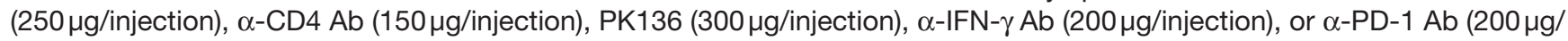
injection), $(\mathrm{n} \geq 7)$ were intraperitoneally injected into mice to deplete $\mathrm{CD} 8^{+} \mathrm{T}$ cells, $\mathrm{CD} 4^{+} \mathrm{T}$ cells, or NK1.1 $1^{+}$cells, neutralize circulating IFN- $\gamma$, or enhance virotherapy with $\alpha-\mathrm{PD}-1 \mathrm{Ab}(\mathrm{P})$, and a log-rank (Mantel-Cox) test was used to compare survival rates $(\mathrm{Q}$ and $\mathrm{R})$, respectively. ${ }^{\star} \mathrm{P}<0.05 ;{ }^{\star \star} \mathrm{P}<0.01$; ${ }^{{ }^{\star \star} \mathrm{P}}<0.001$; and ${ }^{\star \star \star \star} \mathrm{P}<0.0001$. COX-2, cyclooxygenase-2; IFN- $\gamma$, interferon $\gamma$; IL-12, interleukin 12; G-MDSC, granulocytic myeloid-derived suppressor cells; NS, not significant; PBS, phosphate-buffered saline; PFU, plaque-forming units; TGF- $\beta$, transforming growth factor $\beta$; VEGF, vascular endothelial growth factor.

and angiogenesis markers (VEGF and CD105) in tumors after IL-12-armed virus treatment compared with other treatments (figure 3K-M; online supplementary figure $\mathrm{S} 4 \mathrm{G})$. It is interesting that although the IL-12-armed virus treatment did not decrease the TGF- $\beta$ expression in $\mathrm{CD}_{11} \mathrm{~b}^{+}$cells (online supplementary figure $\mathrm{S} 4 \mathrm{H}$ ), it did decrease the TGF- $\beta$ expression in Tregs cells (figure $3 \mathrm{~N}$ ) and $\mathrm{CD} 45^{-}$cells (figure $3 \mathrm{O}$ ), which suggests a potential synergy with PD-1/PD-L1 blockade since the reduction of TGF- $\beta$ signaling in stromal cells can enhance the antitumor immunity of anti-PD-1 antibody treatment. ${ }^{31}$ We further depleted IFN- $\gamma$, NK $1.1^{+}$cells, and $\mathrm{CD}^{+}$and $\mathrm{CD}^{+} \mathrm{T}$ cells by antibodies post vvDDIL-12-FG treatment (figure 3P; online supplementary figure S5) and found that the antitumor effect elicited by wvDD-IL-12-FG treatment was IFN- $\gamma$-dependent and CD8 ${ }^{+}$T-cell-dependent, but not $\mathrm{CD} 4^{+}$T-cell-dependent or NK1.1 $1^{+}$cell-dependent (figure 3Q). Collectively, these data demonstrated that voDD-IL-12-FG treatment, as well as vvDD-IL-12 treatment, tipped the cancer-immune set point in tumor-bearing mice and turned "cold" tumors to "hot" tumors, which significantly extended the survival of mice receiving IL-12-armed virus treatment. Therefore, we hypothesized that the combination with immune checkpoint blockade would enhance the antitumor effects. We previously reported that the anti-PD-1 antibody either alone or combined with vvDD could not elicit an effective antitumor response in the late-stage tumor model. ${ }^{11}$ We tested whether the combination of vvDD-IL-12-FG and anti-PD-1 antibody could improve the therapeutic effects using the latestage tumor model. The survival results showed that in combination with anti-PD-1 antibody, vvDD-IL-12-FG cured all the advanced tumor-bearing mice (figure 3R). Thus, the safe delivery of tethered IL-12 into the TME via oncolytic virus, combined with immune checkpoint 
blockade, makes it possible to revisit IL-12 as a cancer immunotherapy agent. ${ }^{32}$

\section{CONCLUSIONS}

Our data demonstrate that vvDD-IL-12-FG treatment can deliver IL-12 to the tumor bed and tether IL-12 on cell membranes, which is safe and effective in modifying the cancer-immune set point and producing an immunefavorable microenvironment that leads to improved efficacy as a monotherapy. Impressively, in profoundly immunosuppressive, advanced stage disease, vvDD-IL12-FG synergizes with anti-PD-1 antibody therapy, leading to the cure of all late-stage MC38 tumors. Our data suggest that vvDD-IL-12-FG as a new form of IL-12 immunotherapy represents a treatment for cancers that have historically been unresponsive to immune checkpoint blockade-based immunotherapy.

\section{MATERIALS AND METHODS \\ Mice and cell lines}

Female C57BL/6 (B6 in short) and BalB/c mice were purchased from The Jackson Laboratory (Bar Harbor, Maine) and housed in specific pathogen-free conditions in the University of Pittsburgh Animal Facility. Mouse colon cancer MC38-luc, colon cancer CT26-luc, and mesothelioma AB12-luc cells were generated by the infection of parental tumor cells with firefly luciferase-carrying lentivirus and antibiotic blasticidin selection. Normal African green monkey kidney fibroblast CV1, mouse melanoma B16, and Lewis lung cancer cells were obtained from American Type Culture Collection. Primary T cells were grown in RPMI-1640 supplemented with $10 \%$ fetal bovine serum (FBS), $1 \mathrm{mM}$ sodium pyruvate, $2 \mathrm{mM}$ L-glutamine, $10 \mathrm{mM}$ 4-(2-hydroxyethyl)-1-piperazineethanesu lfonic acid (HEPES), $50 \mu \mathrm{M} \beta$-mercaptoethanol, $1 \times$ nonessential amino acid, and $1 \times$ penicillin/streptomycin (Invitrogen, Carlsbad, California). Other cell lines were grown in Dulbecco's modified Eagle's medium (DMEM) supplemented with $10 \%$ FBS, $2 \mathrm{mM}$ L-glutamine, and $1 \times$ penicillin/streptomycin in a $37^{\circ} \mathrm{C}, 5 \% \mathrm{CO}_{2}$ incubator.

\section{Virus generation}

VSC20, a vgf gene-deleted Western Reserve strain vaccinia virus, was used as the parental virus for homologous recombination. Shuttle vectors pCMS1-IRES, pCMS1-IRES-FG, and pCMS1-IRES-RG have been described previously. ${ }^{11}$ Murine IL-12p35 and IL-12p40 cDNAs were inserted into pCMS1-IRES, pCMS1-IRES-FG, or pCMS1-IRES-RG to get shuttle plasmids pCMS1-IL-12p35-IRES-IL-12p40, pCMS1-IL-IL-12p35-IRES-IL-12p40-FG, or pCMS1-IL12p35-IRES-IL-12p40-RG, respectively. The primers for plasmid cloning based on PCR are listed (online supplementary table S1). All these shuttle vectors were used for homologous recombination of murine $I L-12$ variants into the $t k$ locus of the vaccinia viral genome. To make the new viruses vvDD-IL-12, vvDD-IL-12-FG, and vvDD-IL-12-RG,
CV-1 cells were infected with VSC20 at a MOI of 0.1 and then transfected with the shuttle plasmids, resulting in virus mixture. Selection of the new recombinant viruses was based on expression of yellow fluorescent protein in CV1 cells 24 hours post-infection of relative virus mixture. vvDD-YFP, or vvDD for short, a double viral gene-deleted ( $t k-$ and $v g f-$ ) vaccinia virus carrying $y f p$ cDNA at the $t k$ locus, was the control virus for this work.

\section{Viral replication and IL-12 expression in vitro}

MC38-luc $\left(3 \times 10^{5}\right)$, B16 $\left(2 \times 10^{5}\right)$, or AB12-luc $\left(3 \times 10^{5}\right)$ cells were seeded in 24-well plates overnight and infected with vvDD, vvDD-IL-12, vvDD-IL-12-FG, or vvDD-IL-12-RG at a MOI of 1 in $0.15 \mathrm{~mL} 2 \%$ FBS-containing DMEM for 2 hours. Of note, $0.35 \mathrm{~mL} 10 \%$ FBS-containing DMEM was added to cells and the mixture was cultured until harvest at 24 hours post-viral infection. The culture supernatants were harvested to measure IL-12 using ELISA (BD Bioscience, San Jose, California) and the cell pellets were applied either to measure membrane-bound IL-12 using flow cytometry or to extract RNA to measure the viral house-keeping gene $\mathrm{A} 34 \mathrm{R}$ to monitor viral replication and transgene IL-12 expression by RT-qPCR, respectively. To further confirm the membrane association of IL-12, the tumor cells were infected with indicated viruses at MOIs of $0.1,1$, and 5 and harvested 24 hours post-infection to measure membrane-bound IL-12 using ELISA after cleavage of PI-PLC (Sigma, P5542; 8 units per mL PBS).

\section{Cytotoxicity assay in vitro}

Tumor cells were plated at $1.0 \times 10^{4}$ (except B16 cells, which were plated at $5 \times 10^{3}$ ) cells per well in 96-well plates and infected with indicated viruses the next day at different MOIs. Cell viability was determined at 48 hours after infection using CellTiter 96 Aqueous Nonradioactive Cell Proliferation Assay (Promega, Madison, Mississippi) or Cell Counting Kit-8 (Boster Biological Technology, Pleasanton, California).

\section{Primary T cell proliferation assay}

Splenic T cells were isolated from naïve $\mathrm{B} 6$ mice with a pan T cell isolation kit (Miltenyi Biotec, Auburn, CA) and cultured in T-cell medium mentioned above containing $4 \mu \mathrm{g} / \mathrm{mL}$ Con A and $200 \mathrm{U} / \mathrm{mL}$ IL-2 at a density of $2 \times 10^{6} /$ $\mathrm{mL}$ for 2 days $(0.1 \mathrm{~mL}$ per well in 96 -well plates). On the same day, MC38 $\left(3 \times 10^{5}\right)$ cells were seeded in 24-well plates overnight and infected with indicated viruses at an MOI of 5 in $0.15 \mathrm{~mL} 2 \%$ FBS-containing DMEM for 2 hours. And then $0.35 \mathrm{~mL} 10 \%$ FBS-containing DMEM was added to cells and the mixture was cultured until harvest at 24 hours post-viral infection. The mock or virus-infected MC38 cells were harvested and treated with MMC (StressMarq Biosciences: SIH-246) $(200 \mu \mathrm{g} / \mathrm{mL})$ in a $37^{\circ} \mathrm{C}, 5 \% \mathrm{CO}_{2}$ incubator for 2 hours and washed extensively for use. The MMC-inactivated tumor cells were resuspended in T-cell medium at a density of $1 \times 10^{7} / \mathrm{mL}$ per well and $0.1 \mathrm{~mL}$ was added to T-cell-containing 96 -well 
plates if needed. For some wells, MMC-inactivated cells were pre-incubated with anti-mIL-12 antibody $(5 \mu \mathrm{g} / \mathrm{mL}$; BioLegend: \#505304) for half an hour before coculture. The proliferation of activated $\mathrm{T}$ cells was measured using MTT assay 2 days after coculture.

\section{Rodent tumor models}

B6 mice were intraperitoneally inoculated with $5 \times 10^{5}$ MC38-luc cancer cells or BalB/c mice were intraperitoneally inoculated with $4 \times 10^{5} \mathrm{AB} 12$-luc or CT26-luc cancer cells, respectively, and divided into required groups at the indicated day post-tumor cell inoculation according to tumor size based on live animal IVIS imaging, performed using a Xenogen IVIS 200 Optical In Vivo Imaging System (Caliper Life Sciences, Hopkinton, Massachusetts). Grouped mice were intraperitoneally injected with indicated viruses, antibodies, the combinations, or PBS. In some experiments, anti-CD8 Ab (clone 53-6.7; Bio X Cell; $250 \mu \mathrm{g} /$ injection), anti-CD4 Ab (clone GK1.5, Bio X Cell; $150 \mu \mathrm{g} /$ injection), anti-NK1.1 Ab (clone PK136, Bio X Cell; $300 \mu \mathrm{g} /$ injection), or anti-IFN- $\gamma$ Ab (clone XMG1.2, Bio X Cell; $200 \mu \mathrm{g} /$ injection) were intraperitoneally injected into mice to deplete $\mathrm{CD} 8^{+} \mathrm{T}$ cells, $\mathrm{CD} 4^{+}$ $\mathrm{T}$ cells, or NK1.1+ cells or neutralize circulating IFN- $\gamma$, respectively. Anti-PD-1 Ab (clone RMP1-14; Bio X Cell; $200 \mu \mathrm{g} /$ injection) was intraperitoneally injected into mice for combination therapy. In some experiments, mice were sacrificed 5 days after treatments to harvest peritoneal tumor nodules for further analysis. In some experiments, mice were sacrificed to harvest spleens 8 days after treatments and splenocytes $\left(4 \times 10^{5}\right)$ were isolated and restimulated with MMC-inactivated MC38 cells $\left(4 \times 10^{4}\right.$ cells $)$ in $200 \mu \mathrm{L}$ T-cell medium mentioned above at $37^{\circ} \mathrm{C}, 5 \%$ $\mathrm{CO}_{2}$ for 2 days. The concentration of IFN- $\gamma$ in the culture supernatants was measured by ELISA. MC38-luc-tumorbearing B6 mice treated with vvDD-IL-12-FG, which had survived for more than 250 days, were subcutaneously challenged with $5 \times 10^{5}$ MC38 or $1 \times 10^{6}$ Lewis lung cancer cells per mouse. Naïve B6 mice also received the same dose tumor challenge as a control. Subcutaneous tumor size was measured using an electric caliper in two perpendicular diameters.

\section{Assessment of treatment-related toxicity}

MC38-luc-bearing B6 mice receiving virus treatment at day 9 after tumor inoculation with the viral dose of $5 \times 10^{8}$ or $1 \times 10^{9} \mathrm{PFU} /$ mouse were used to monitor the toxicity. Mouse blood samples were collected daily from mocktreated or virus-treated mice and kept for 2 hours at room temperature and sera were separated by centrifugation. These mice were sacrificed at day 5 after treatments to collect blood, tumor nodules, lungs, and kidneys. Tumor nodules were weighed, homogenized in lysis buffer (RayBiotech, CODE: EL-lysis) in the presence of protease inhibitor cocktail (Sigma, \#111836170001) at $10 \mu \mathrm{L}$ lysis buffer per milligram tumor using a FastPrep Cell Disrupter (Model FP120) (Qbiogene, Carlsbad, California), and centrifuged to collect supernatant. Tumoral supernatant and sera were used to measure IL-12 and IFN- $\gamma$; sera were also used to measure ALT and AST, using commercialized kits (BioLegend and G-Biosciences, respectively), according to the vendors' instructions. Water content was used to monitor tissue edema. Briefly, wet tissue was weighed and dehydrated overnight over $100^{\circ} \mathrm{C}$ in a chemical fume hood. The weight difference between wet tissues and dry tissues was calculated.

\section{Flow cytometry}

Collected tumor tissues were weighed and incubated in RPMI 1640 medium containing 2\% FBS, $1 \mathrm{mg} / \mathrm{mL}$ collagenase IV (Sigma: \#C5138), $0.1 \mathrm{mg}$ hyaluronidase (Sigma: \#H6254), and 200U DNase I (Sigma: \#D5025) at $37^{\circ} \mathrm{C}$ for 1-2 hours to make single cells. In vitro virus-infected cells or single cells from tumor tissues were blocked with $\alpha-\mathrm{CD} 16 / 32 \mathrm{Ab}$ (clone 93, eBioscience: \#14-0161-85; $1: 1000)$ and then stained with antibodies against mouse CD45 (PerCP-Cy5.5 or FITC, clone: 30-F11, BioLegend: \#103132 or 103108; 1:300), CD4 (APC, clone: RM4-5, eBioscience: \#17-0042-81; FITC, clone: GK1.5, BD Biosciences: \#553729, 1:300), Foxp3 (PE, clone: FJK16s, eBioscience: \#12-5773-82; 1:100), CD8 (PE or APC, clone: 53-6.7, eBioscience: \#12-0081-85 or 17-0081-83; 1:300), PD-1 (PerCP-Cy5.5 or Brilliant Violet 785, clone: 29F.1A12, BioLegend: \#135208 or \#135225; 1:300), TIM-3 (Biotin-TIM-3, clone: RMT3-23, BioLegend: \#119720; 1:300+PE SA, eBioscience: \#12-4317-87; 1:1000), TIGIT (Biotin-TIGIT, clone: 1G9, BioLegend: \#142113; 1:300+PESA, eBioscience: \#12-4317-87; 1:1000), LAG-3 (PE, clone: C9B7W, BioLegend: \#125208; 1:300), CD11b (FITC or PE, clone: M1/7, BioLegend: \#101 206 or \#101208; 1:300), Ly-6G (APC, clone: 1A8, eBioscience: \#17-9668-82; 1:300), Ly-6C (FITC, clone: HK1.4, BioLegend:\#128006; 1:300), PD-L1 (APC, clone: 10F.9G2, BioLegend: \#124312, 1:300), TGF- $\beta$ (Brilliant Violet 421, clone: TW7-16B4, BioLegend: \#141408, 1:100), and IL-12p40 (PE, clone: C17.8, eBioscience: \#12-7123-82; 1:300). The intracellular staining kit for Foxp3, TGF- $\beta$, and IFN- $\gamma$ staining was purchased from BioLegend. Samples were collected on a BD Accuri C6 cytometer or BD LSR Fortessa I, and data were analyzed using BD Accuri C6 cytometer software or FlowJo software (Tree Star Inc., Ashland, Oregon).

\section{RT-qPCR}

Total RNA was extracted from viral-infected cells or tumor tissues using the RNeasy Kit (Qiagen, Valencia, California). One microgram of RNA was used for cDNA synthesis, and 25 to $50 \mathrm{ng}$ of subsequent cDNA was used to conduct mRNA expression TaqMan analysis on the StepOnePlus system (Life Technologies, Grand Island, New York). All primers for the analysis were purchased from Thermo Fisher Scientific (Waltham, Massachusetts). Gene expression was normalized to the housekeeping gene HPRT1 and expressed as fold increase $\left(2^{-\triangle \mathrm{CT}}\right)$, where $\Delta \mathrm{CT}=\mathrm{CT}_{\text {(target gene) }}-\mathrm{CT}{ }_{(\text {HPRT1) }}$. 


\section{Statistics}

Statistical analyses were performed using unpaired Student's t-test (GraphPad Prism V.7). Data are means \pm SD. Animal survival is presented using Kaplan-Meier survival curves and was statistically analyzed using a log-rank test (GraphPad Prism V.7). Values of $\mathrm{p}<0.05$ were considered statistically significant, and all $\mathrm{p}$ values were two-sided. In the figures, standard symbols are used: $* \mathrm{p}<0.05 ; * \mathrm{*}<0.01$; $* * * \mathrm{p}<0.001 ;$ and $* * * * \mathrm{p}<0.0001$.

\section{Author affiliations}

${ }^{1}$ Department of Surgery, University of Pittsburgh, Pittsburgh, PA, USA

${ }^{2}$ UPMC Hillman Cancer Center, Pittsburgh, PA, USA

${ }^{3}$ Department of Immunology, School of Biology and Basic Medical Sciences, Medical College, Soochow University, Suzhou, Jiangsu Province, China

${ }^{4}$ Cancer Center, Union Hospital, Huazhong University of Science and Technology, Wuhan, Hubei Province, China

${ }^{5}$ Xiangya School of Medicine, Central South University, Changsha, Hunan Province, China

${ }^{6}$ Shanghai Jiao Tong University Affiliated Sixth People's Hospital, Shanghai, China

${ }^{7}$ Department of Cancer Center, Renmin Hospital of Wuhan University, Wuhan, Hubei Province, China

${ }^{8}$ Department of Oncology and Hematology, China-Japan Union Hospital of Jilin University, Changchun, Jilin Province, China

Acknowledgements The authors thank Christine Burr, BA (Scientific Writer, Department of Anesthesiology and Perioperative Medicine/Department of Surgery, University of Pittsburgh School of Medicine) for her editorial assistance with the manuscript.

Contributors YG, HW, JR, WL, LC, HC, JY, ED, CM, and ZL conducted the experiments and collected the data; ZL, ZSG, and DLB conceived the project; ZL and DLB designed the experiments, interpreted the data, and wrote the manuscript. SJ provided advice and made suggestions for the manuscript.

Funding This project was supported by UPMC funds (Retention funds and ITTC funds) and used UPMC Hillman Cancer Center shared facilities supported in part by NIH grant award P30CA047904.

Competing interests The University of Pittsburgh has filed a patent application directed to the subject matter of this manuscript.

\section{Patient consent for publication Not required.}

Ethics approval The animal studies have been approved by the Institutional Biosafety Office, and Institutional Animal Care and Use Committee at the University.

Provenance and peer review Not commissioned; externally peer reviewed.

Data availability statement The data used or analyzed during this study are included in this article and available from the corresponding authors upon reasonable request.

Open access This is an open access article distributed in accordance with the Creative Commons Attribution Non Commercial (CC BY-NC 4.0) license, which permits others to distribute, remix, adapt, build upon this work non-commercially, and license their derivative works on different terms, provided the original work is properly cited, appropriate credit is given, any changes made indicated, and the use is non-commercial. See http://creativecommons.org/licenses/by-nc/4.0/.

\section{REFERENCES}

1 Chen DS, Mellman I. Elements of cancer immunity and the cancerimmune set point. Nature 2017;541:321-30.

2 Chen DS, Mellman I. Oncology meets immunology: the cancerimmunity cycle. Immunity 2013;39:1-10.

3 Sharma P, Hu-Lieskovan S, Wargo JA, et al. Primary, adaptive, and acquired resistance to cancer immunotherapy. Cell 2017;168:707-23.

4 Hodi FS, O'Day SJ, McDermott DF, et al. Improved survival with ipilimumab in patients with metastatic melanoma. $N$ Engl J Med 2010;363:711-23.

5 Topalian SL, Hodi FS, Brahmer JR, et al. Safety, activity, and immune correlates of anti-PD-1 antibody in cancer. $N$ Engl J Med 2012;366:2443-54.
6 Zou W, Wolchok JD, Chen L, et al. Pd-L1 (B7-H1) and PD-1 pathway blockade for cancer therapy: mechanisms, response biomarkers, and combinations. Sci Transl Med 2016;8:328rv4.

7 Gajewski TF. The next hurdle in cancer immunotherapy: overcoming the non-T-cell-inflamed tumor microenvironment. Semin Oncol 2015;42:663-71.

8 Bartlett DL, Liu Z, Sathaiah M, et al. Oncolytic viruses as therapeutic cancer vaccines. Mol Cancer 2013;12:103.

9 Bommareddy PK, Shettigar M, Kaufman HL, et al. Integrating oncolytic viruses in combination cancer immunotherapy. Nat Rev Immunol 2018;18:498-513.

10 Pearl TM, Markert JM, Cassady KA, et al. Oncolytic virus-based cytokine expression to improve immune activity in brain and solid tumors. Mol Ther Oncolytics 2019;13:14-21.

11 Liu Z, Ge Y, Wang H, et al. Modifying the cancer-immune set point using vaccinia virus expressing re-designed interleukin-2. Nat Commun 2018;9:4682.

12 Colombo MP, Trinchieri G. Interleukin-12 in anti-tumor immunity and immunotherapy. Cytokine Growth Factor Rev 2002;13:155-68.

13 Atkins MB, Robertson MJ, Gordon M, et al. Phase I evaluation of intravenous recombinant human interleukin 12 in patients with advanced malignancies. Clin Cancer Res 1997;3:409-17.

14 Leonard JP, Sherman ML, Fisher GL, et al. Effects of single-dose interleukin-12 exposure on interleukin-12-associated toxicity and interferon-gamma production. Blood 1997;90:2541-8.

15 Cohen J. IL-12 deaths: explanation and a puzzle. Science 1995;270:908.

16 Nagarajan S, Selvaraj P. Glycolipid-Anchored IL-12 expressed on tumor cell surface induces antitumor immune response. Cancer Res 2002;62:2869-74.

17 Poutou J, Bunuales M, Gonzalez-Aparicio M, et al. Safety and antitumor effect of oncolytic and helper-dependent adenoviruses expressing interleukin-12 variants in a hamster pancreatic cancer model. Gene Ther 2015;22:696-706.

18 Pan W-Y, Lo C-H, Chen C-C, et al. Cancer immunotherapy using a membrane-bound interleukin-12 with B7-1 transmembrane and cytoplasmic domains. Mol Ther 2012;20:927-37.

19 Abastado J-P. The next challenge in cancer immunotherapy: controlling T-cell traffic to the tumor. Cancer Res 2012;72:2159-61.

20 Zhang L, Morgan RA, Beane JD, et al. Tumor-Infiltrating lymphocytes genetically engineered with an inducible gene encoding interleukin-12 for the immunotherapy of metastatic melanoma. Clin Cancer Res 2015;21:2278-88.

21 Wang P, Li X, Wang J, et al. Re-designing interleukin-12 to enhance its safety and potential as an anti-tumor immunotherapeutic agent. Nat Commun 2017;8:1395.

22 McCart JA, Ward JM, Lee J, et al. Systemic cancer therapy with a tumor-selective vaccinia virus mutant lacking thymidine kinase and vaccinia growth factor genes. Cancer Res 2001;61:8751-7.

23 Zeh HJ, Downs-Canner S, McCart JA, et al. First-in-man study of Western reserve strain oncolytic vaccinia virus: safety, systemic spread, and antitumor activity. Mol Ther 2015;23:202-14.

24 Downs-Canner S, Guo ZS, Ravindranathan R, et al. Phase 1 study of intravenous oncolytic poxvirus (VvDD) in patients with advanced solid cancers. Mol Ther 2016;24:1492-501.

25 Chen X, Zaro JL, Shen W-C, et al. Fusion protein linkers: property, design and functionality. Adv Drug Deliv Rev 2013;65:1357-69.

26 Tugues S, Burkhard SH, Ohs I, et al. New insights into IL-12mediated tumor suppression. Cell Death Differ 2015;22:237-46.

27 Naik AM, Chalikonda S, McCart JA, et al. Intravenous and isolated limb perfusion delivery of wild type and a tumor-selective replicating mutant vaccinia virus in nonhuman primates. Hum Gene Ther 2006;17:31-45.

28 Fortin $\mathrm{C}$, Huang $\mathrm{X}$, Yang $\mathrm{Y}$, et al. Nk cell response to vaccinia virus is regulated by myeloid-derived suppressor cells. J Immunol 2012;189:1843-9.

29 Cao X, Leonard K, Collins LI, et al. Interleukin 12 stimulates IFN-gamma-mediated inhibition of tumor-induced regulatory T-cell proliferation and enhances tumor clearance. Cancer Res 2009;69:8700-9.

30 Ribas A. Adaptive immune resistance: how cancer protects from immune attack. Cancer Discov 2015;5:915-9.

31 Mariathasan S, Turley SJ, Nickles D, et al. Tgf $\beta$ attenuates tumour response to $P D-L 1$ blockade by contributing to exclusion of $T$ cells. Nature 2018;554:544-8.

32 Berraondo P, Etxeberria I, Ponz-Sarvise M, et al. Revisiting interleukin-12 as a cancer immunotherapy agent. Clin Cancer Res 2018;24:2716-8. 